\title{
7. Artificial Intelligence and the Temporality of Machine Images
}

\author{
Andrew R. Johnston
}

\begin{abstract}
DeepMind, a recent artificial intelligence technology created at Google, references in its name the relationship in AI between models of cognition used in this technology's development and its new deep learning algorithms. This chapter shows how AI researchers have been attempting to reproduce applied learning strategies in humans but have difficulty accessing and visualizing the computational actions of their algorithms. Google created an interface for engaging with computational temporalities through the production of visual animations based on DeepMind machine-learning test runs of Atari 2600 video games. These machine play animations bear the traces of not only DeepMind's operations, but also of contemporary shifts in how computational time is accessed and understood.
\end{abstract}

Keywords: artificial intelligence, animation, digital media, video games, technology, history

DeepMind, a recent artificial intelligence technology created at Google, references in its name the relationship in AI between models of cognition used in this technology's development and the deep learning algorithms that make up its layers of nonlinear processing units. With DeepMind Google has been attempting to reproduce patterns of visually based reinforcement learning strategies observed in human subjects through the use of big data and probability scenarios in neural networks. Like other AI that were created and used in speech recognition or machine translation applications, researchers at Google encountered two problems: 1) how to model reactive sensory systems and 2) how to visualize and access

Volmar, A. and K. Stine (eds.), Media Infrastructures and the Politics of Digital Time: Essays on Hardwired Temporalities. Amsterdam: Amsterdam University Press, 2021 DOI 10.5117/9789463727426_CHO7 
their mechanics when the systems operate below thresholds of human sensory perception and cognition. The second question is one endemic to contemporary computational processes and sets of big data generally, whose weighty flows of indeterminate scales of information are born out through representational tropes or descriptions that refer to their inaccessibility. Researchers managed both problems by designing an AI system that could train to win emulated Atari 2600 video games such as Qbert, Space Invaders, Breakout, and 46 others, each played 600 times in order to visualize the mechanics of the AI. The gameplay videos, or machine runs, of these training sessions offer representational data points for researchers who map how reinforcement learning strategies are employed over the course of various gameplay scenarios, revealing in visual space how the AI adapts to different contexts and evaluation criteria through operations in time that are otherwise inaccessible.

This out of reach character of digital technologies is commonplace. Materialist analyses of digital media, like the ones included in this volume, have brought attention to the disjuncture of temporalities present in contemporary technological systems and regimes of experience. Running at speeds faster than that which sensation can detect and through machinations outside the purview of consciousness, digital technology seems to break traditions of technological synchronization that match human experience to the temporal actions of machines. Descriptions of this historically antecedent organization of time and technics can be seen in the work of Mary Ann Doane, who, following E.P. Thompson, argues that a nineteenth century reorganization of time was engendered by industrial revolution machines and clocks that enabled it to be measured and divided. This also externalized temporality as a "surface phenomenon" that individuals attempted to capture through mediated representations. ${ }^{1}$ That chase was precisely what Paul Virilio worried over, as he envisioned how the mutual accelerations of technology and understandings of time could erode the limits of human history, knowledge claims, and order. $^{2}$

The link between human sensation and machinic temporality is what scholars such as Mark Hansen and Wolfgang Ernst argue has been severed by contemporary digital technologies. Ernst emphasizes how digital technologies materially instantiate a time outside of consciousness through the calculation and flow of signals so that even digital "storage is nothing but a limit value of transfer ... storage is a transfer across a temporal distance." This articulation of time and space is microscopic in scale and employed by digital media for the abstractions of signal transmissions, which allow for later formal actions that are made available to the senses by these objects. 
Hansen makes a similar point, arguing that twenty-first-century media, or digital objects and network infrastructures, "operate predominantly, if not almost entirely, outside the scope of human modes of awareness (consciousness, attention, sense perception, etc.)."4 The resulting human experiences with these media and their environments are therefore only indirect relative to their machinations; since one cannot witness their signal pulses, to experience them is to be at a remove from their technical work. Hansen argues that because of this arrangement contemporary digital media has a predictive relation to our bodies in a feed forward architecture "that literally mediate the data of causal efficacy (as measured, calculated, and analyzed by twenty-first-century media) for future consciousness to factor into its activity-to-come." Since digital technologies are built upon indirect engagements with their inner workings, their signal transmissions are structured through predictions of future action in a race with consciousness and experience to produce illusions of interactivity.

All these epistemological formulations are rooted in contemporary digital media. And for good reason, since digital technology used for computer graphics or other applications in the 1960 s and 1970s was notoriously slow relative to analog computers and often relied on paper storage. ${ }^{6}$ But the above authors also emphasize how the operations of contemporary digital media are at such a remove that they appear opaque, a black boxing of technology that Ernst, as well as Bruno Latour, warn against in understanding material renderings of experience brought about by shifting technical regimes. ${ }^{7}$ The seeming opacity of digital media is certainly a cultural phenomenon that generates anxiety, often manifest in new genres or mediated by horror narratives, arguments persuasively made by scholars such as James J. Hodge, Shane Denson, and Adam Hart. ${ }^{8}$ That said, this perspective is not totalizing and, as the strange periodizing of Hansen and Ernst reveal, is a shifting process born of multiplicity and contradiction rather than singularity and uniformity. As Ernst emphasizes, "a media-archaeological view of the temporal modes of media almost inevitably leads to a critique of the totalizing collective singularity of 'time' itself."9

To perform this media archeological analysis is to open the black boxes of contemporary digital technologies that can appear so opaque. Doing so performs an epistemological reverse engineering that makes manifest the impact of technologies in the production of knowledge through their mechanisms of sensual and information presence. ${ }^{10}$ And it also clarifies the contours of that epistemology and mediations of temporal experience. While the phenomenal actions of computational media may index obscurity, they also point towards and create new arrangements 
and networks of activity. Such changes may induce anxiety in cultural narratives, a reaction found during other periods of shifting temporalities born of technological change, exemplified in Alvin Toffler's 1970 book Future Shock. ${ }^{11}$ But as past studies of these moments have shown, such transitions are often fractured with multiple layers of technicity interwoven with one another to both manage these changes and develop use strategies. ${ }^{12}$ In this light the machine run videos of DeepMind's Atari play have particular significance, since they are not created as a formal articulation that gestures towards an unknowable obscurity, but instead attempts to quantify and abstract the temporal and computational actions of the AI for later modification. In Google's published work, the abstract neural network of DeepMind is displayed with corresponding screenshots from gameplay to provide illustrations of what they argue is machine learning. These gameplay animations index machine action and are a means through which Google can refine and develop computational deep learning. Doing so, I argue, positions animation as an epistemic object and tool to make nonhuman cognition and time sensually present. Functioning as an investigatory device, it maintains the aesthetic wonder that animation is often associated with, but simultaneously reveals different technical operations buried in the AI system that produce these articulations. ${ }^{13}$ These unseen microtemporal actions are animations unto themselves, since the system analyzes, abstracts, and then synthesizes images into taxonomic frameworks or movements acted upon in a different layer within its feed forward architecture. Though operating at different scales of time, the qualities of technical abstraction and synthesis in other animations are still present, though addressed for a system whose visual syntheses have been modeled from human perception and that we cannot see. But these actions can also become the means through which phenomenal images are generated that then makes sensually present information for human users, a secondary animation layered onto the others. I argue that together this stack of animation produces the interface for working with and manipulating networked infrastructures of AI, an interweaving of what Ernst, quoting Gilbert Simondon, calls different levels of temporal knowledge: "a micro-level of physical and techno-physical processes, [and] a meso-level of psychic-cognitive processes." ${ }^{{ }^{114}}$ Layering them onto one another both enables speculative knowledge about the AI system's temporal modulations and about our relation to these new actions of time. The remainder of this essay will work through and open this AI interface, moving up and down its stacks to reveal animation's role in the diverging temporalities that mark these technologies and this moment. 
AI emerged as a field in the 1950s out of cybernetics and one of its earlier and most long-lasting research areas was visual pattern classification and the development of machines that would have the ability to detect predetermined objects within given fields. Though operating in fits and starts over several decades, this area of AI has recently become a center of inquiry, in part due to the accelerated growth of graphics processing units used in feed forward neural networks created after what many call an AI winter in research during the 1970s. ${ }^{15}$ Unlike other AI information oriented platforms, DeepMind has action-oriented goals achieved through rewards defined within specific environments. It is designed to create software agents that "take actions in an environment so as to maximize some notion of cumulative reward." ${ }^{\prime 6}$ Taken as a whole, this is a feedback system that explores and modifies the agent's environment and like earlier AI systems uses behaviorist psychology frameworks. But its modeling of the ways individuals solve problems through feedback mechanisms is put in conjunction with a hierarchical sorting and categorization of sensory data. Previous AI systems were built around applications set in motion by particular stimuli. IBM's Watson, for instance, is a natural language and information retrieval system, whose functions would be triggered by spoken questions. ${ }^{17}$ Because of this, the system does not explore or test its environment through sensory data, but instead operates through an if-then Boolean logic so that if particular inputs exist, then a functioning action within the platform will result. Using a behavior tree for executed actions, the platform is dependent upon language, accent, and the information database accessible to it.

The exploration of an environment through visual data was still the most prized area of AI research and much of its success has emerged from investigations into vision performed by Nobel prize winning neurobiologists David Hubel and Torsten Wiesel. Beginning in 1958 Hubel and Wiesel traced the mechanisms of action within the visual cortex and showed how the brain processes visual information from the retina through a columnar architecture filled with simple and complex cells, each of which is sensitive to particular visual features for specific parts of the visual field. ${ }^{18}$ In their experiments they determined how the visual cortex of a cat, and later other animals, uses simple nerve cells to locate edges or borders of contrast along with other formal features and that the cortex employs complex nerve cells to combine input from simple cells to gauge motion and other actions. Finally developing a topographical map of the visual cortex, Hubel and Wiesel showed how different neurons from the retina operate as inputs to produce binocular perception and the visual pathway of information in the brain. ${ }^{19}$ 
Importantly, Hubel and Wiesel's experiments also illustrated the feed forward mechanisms through which visual perception functions, which rather than having a singular feedback loop for input and outputs, nests multiple layers of inputs and outputs that don't cycle or loop back to each other but move in a linear fashion. Fundamentally, the nodes in this system constantly evaluate or categorize the input they receive, each making slight transformations before outputting to the next node in a unidirectional manner through time. This design became the model for large computational feed forward networks, which were successfully employed to analyze and categorize images by computer scientists like Yann LeCun in the late 1980s. He developed convolutional neural networks that had many filter layers for the categorization and identification of image features, enabling the system to work through the logic and pattern differentiation problems that plagued AI image research in the past. This requires using Hubel and Wiesel's analysis of edge detection for images but in a large enough network that the subsampling of images continues until the system can accurately identify objects. Breaking the images up many times over, the network races to abstract the image, compare those pieces to others it has analyzed before, and then categorize and take action on that decision. Citing the neurobiologists as a precedent, LeCun describes how this convolutional neural network can be used for handwriting and optical character recognition machine learning, each of which has been employed by the US postal service and several banking institutions. ${ }^{20}$ Google's DeepMind team references and builds from LeCun's model for "exploiting the local spatial correlations present in images" in order to take into account viewpoints that the AI employs while exploring visual space. ${ }^{21}$ Once again, for DeepMind, the AI does not just seek particular data from sensory inputs, but attempts to organize all the information presented to its inputs within a specified domain of action. It explores but categorizes according to specific criteria. Like other contemporary AI systems, DeepMind is a sophisticated bot technology and is an object categorization machine using a feed forward network agent. All the visual information presented to the AI is sorted as quickly as it can through its matrix of subsampling nodes in order to then provide actionable information within the machine's environment.

For those designing AI, the speed of this sorting is the problem. Experiments with machines that could perform these actions took place almost immediately after Hubel and Wiesel's experiments. In the late 1950s Frank Rosenblatt created a pattern classification algorithm called a perceptron that attempted to categorize visual images. Put simply, the perceptron would 
evaluate image information presented to it and determine, through if-then logical statements, whether or not the image had features of a preassigned category. By utilizing multiple inputted weights, or parts of an image, the algorithm could examine the image several times to compare these weighted values to one another in the overall assessment of the image as belonging or not belonging to the pre-assigned category. After a number of tries, certain inputs are deemed more important than others in the image for determining whether or not it belongs to a category, such as "cat pictures." ${ }^{22}$ At the time, the adaptive quality of the algorithm's operations produced massive speculation about the future of AI in the popular press, especially after Rosenblatt created an analog computer for the perceptron that employed 400 photocells for inputs whose resistance parameters were controlled by motors. ${ }^{23}$ Thinking he had successfully determined whether a tank was in a photograph or not, an application sought after by the U.S. military, Rosenblatt postulated that his machine had "original ideas" and that "as a concept, it would seem that the perceptron has established, beyond doubt, the feasibility and principle of nonhuman systems which may embody human cognitive functions." ${ }^{24}$ This fantasy, however, was short-lived since Rosenblatt was actually guessing at what his machine was classifying, learning later that the perceptron was not identifying a tank in photographs, but rather the time of day. ${ }^{25}$ For Rosenblatt, not having an interface through which he could make phenomenal the microtemporal actions of the machine resulted in not knowing what the machine could or would do, a blindness to both its rhythms of time and its output of information.

Animation solves this problem by both completing a visualized feedback loop for researchers and projecting fantasies of autonomy onto its actions in an environment. But the question of reinforcement learning and its relation to mediations of animation loomed large for Google's research team, since DeepMind both responds to and changes the environmental conditions through which it receives sensory information. In short, Google wanted to display an interface of time through animation so it could show consequences of computational temporalities and their effect on a phenomenal world. In attempting to develop this behaviorist project, Google isolated the visual input available for the system and its actionable output. Using emulations of the Atari 2600 games, they coded DeepMind's sensory inputs to be an $84 \times 84$ pixel gameplay grid and game scores, the latter of which would function as evaluation criteria. Structuring the inputs this way not only isolates ideas of reinforcement learning and processing to visual systems, but also limits specific visual operations, namely motion 
detection. Google wanted to develop an AI algorithm in DeepMind that could be used on large neural networks and therefore isolated various parameters of the system at its genesis. Emphasizing a particular action in reinforcement learning, DeepMind excels when encountering puzzles or obstacles that require a measured degree of planning. As Google explains, in Breakout "the agent learns the optimal strategy, which is to first dig a tunnel around the side of the wall allowing the ball to be sent around the back to destroy a large number of blocks" but also admits that "games demanding more temporally extended planning strategies still constitute a major challenge." ${ }^{26}$ Throughout their description of DeepMind it becomes clearer as to why Google selected Atari 2600 games. Not only is the game environment limited spatially and temporally, but the Atari's sprites are also abstract objects with clear figure/ground relations whose spatial correlations require less processing than other platforms. Such reductions focalize the action of the AI while also conflating the rules or logics of each game with the mechanics of the system, an operation that Stephanie Boluk and Patrick LeMieux identify as prevalent within video games. ${ }^{27}$ To be clear, it is the mechanics of the specific game that DeepMind categorizes, since the system cannot abstract the principles of gameplay from one game to another, but instead must begin again and learn the design logics and operations of each game from scratch. The researchers functionally bar the AI from accessing the code and other algorithmic functions that produce the Atari games, a more efficient means through which the neural network could learn the inputs and outputs associated with this gameplay. Instead, they develop an artificial barrier that attempts to mimic a naive player, a sort of DeepMind noob, whose ultimate precociousness is revealed in a dazzling technological display. It is a brilliant marketing move, but also shows how the $\mathrm{AI}$ is learning the mechanics available to objects in the game more than learning how to "play." Google's conflation, like the one Boluk and LeMieux identifies, of video game logic with object mechanics, nonetheless produces a projection of mastery onto the machine as it sorts through various values, actions, and temporal relations over the 600 iterations of play for each Atari game. Since the AI operates through conditions of gameplay dependent upon display projections, the resulting actions run as animations and visually mark the system's performance through the game's challenges.

As such, the animations that make present the operations of the AI exist within a well-known genre that performs a similar function: tool-assisted speed runs. These are programs that exploit the serial interface of emulated video games to reveal the most efficient combination of inputs into the 
game to finish or "win" it. Through an almost linear editing of possible commands within the game's interface, a recorded animation of action emerges and makes phenomenal the operations of the software. Creating an AI interface like this makes familiar both unseen temporalities of the system as well as the aesthetic and informational form of its output for people familiar with the Atari games. The animations make relatable the AI's operations while acutely marking its differences from human users. Though competitive speed runs performed by human players have been popular within gaming communities for some time, the contrast of these with tool-assisted speed runs, as LeMieux explains, more generally "reveals the alienating effects of digital seriality and dramatizes the distinction between human and machine scales of temporality. ${ }^{28}$ With DeepMind, the alienation builds over time since the spectacular failure of the $\mathrm{AI}$ is put on display over the first hundred iterations of gameplay. By limiting the AI's inputs to the screen and score, Google blinds DeepMind to the serial interface of the Atari games, but simultaneously creates visual access to DeepMind's processing operations for researchers. In this way, the machine run animations function as a heuristic tool within the laboratory, standing in not only for ideal human cognition in reinforcement learning scenarios, but modulations of time as well. Google's comparison of gameplay between the $\mathrm{AI}$ and professional game testers emphasize this point, showing how in games like Road Runner, Star Gunner, or Assault, DeepMind operates at or above what it characterizes as "human-level" gameplay, boasting that it "performs at a level that is broadly comparable with or superior to a professional human games tester ... in the majority of games." ${ }^{29}$ Thus, through DeepMind's limited ocular mimicry to analyze its reinforcement learning architecture, the resulting animations bridge forms of human and nonhuman subjectivity by manifesting phenomenal, aesthetic action that both subjects could produce.

Again, without losing its aesthetic contours, animation in this context becomes an instrument of speculation, or what historians of science describe as an epistemic object: an experimental form characterized by indeterminacy within a historical field, but whose instantiations mark patterns of emerging knowledge and categories. Karin Knorr Cetina calls them "objects of knowledge [that] appear to have the capacity to unfold indefinitely ... like open drawers filled with folders extending indefinitely" since their technical composition is in a constant state of change..$^{30}$ Epistemic objects' changes speak to historic patterns that are dependent upon knowledge formations. Like Lisa Gitelman's examination of documents as having a know-show function, that is, of having "the kind of knowing that is wrapped up with 
showing, and showing wrapped up with knowing," animation here works in the same way: as a means of knowing the temporal modulations buried in the AI's operations, and showing the ways in which these machinic temporalities interface with human cognition and sensation. ${ }^{31}$ No longer printing results like computational outputs of the past, $\mathrm{AI}$ and contemporary computer science research uses animation to index and explore interfaces of time. By making sensually apparent the temporal vectors of processing in DeepMind and pitting those against familiar logics of time in game mechanics, Google manifests a laboratory of AI time, acknowledging that as they develop their algorithms, they will turn to games with "more temporally extended planning strategies."

Positioning animation as tool of knowledge production stacked into an interface with multiple directions of address aimed at nonhuman and human actors punctuates its importance in contemporary digital culture. As Hodge emphasizes relative to the historicity of digital media, "animation captures the time-based volatility of digital media" and reveals forms of historical experience constituted through digital technology's obscured articulations of time. ${ }^{32}$ Similarly, the stacks of animation in AI constellates another point in animation's history of the ways in which humans and nonhumans are suffused or intertwined in its constitution, even when seemingly separate from a distance as in the animations of DeepMind's machine runs. Google's animations serve as a means of assessment for DeepMind, a practice which, as they allude to above, has continued in the development of this AI into new gameplay arenas and platforms, such as Starcraft II. ${ }^{33}$ Inviting professional Starcraft II game players to compete against DeepMind, the uncertainty and anxiety over the system's refined iterations is captured not only by the screen recordings of gameplay, but also by reaction shots of DeepMind's development team. Functioning as an epistemological agent, animation for these researchers offers the best means through which traces of the algorithmic functions of sorting and categorizing can be found. It is a communicative medium that indexes an action rhetorically compared to human intelligence and projected as being autonomous, or seemingly alive. This sense of independent action that these machine runs take, and that so much visually based AI has, is a legacy of equivalences of sight with mastery and intelligence, a story often rehearsed and recently challenged by many. ${ }^{34}$ As Bernard Geoghegan illustrates, by the mid-twentieth century this visual emphasis had become fully integrated into both cybernetics and the development of computer visualization systems. ${ }^{35}$ This led to the development of AI that could, in the popular press, make claims to possessing forms of intelligent learning because of actions built 
around the identification, categorization, and analysis of objects within puzzle environments.

Visualizing these actions and making them seen through an interface was equally as important. All the projections of intelligence and senses of life onto DeepMind's animations are not simply bound to the moving images, but also to the technical artifacts that generate them, whose mechanisms seemingly contain an energy that produces actions. By creating a phenomenal action of DeepMind's reinforcement learning processes, Google's research team both employ and index an articulation of animation defined through its ability to make visible modulations of time within contemporary digital media. Its stacks contain prisms of time, with multiple addresses, but these technical articulations make intelligible digital networks while projecting fantasies of agency onto them. The wonder of animation, its projection of movement that theorists like Sergei Eisenstein and Alan Cholodenko have characterized as lifelike, becomes a means of making visible algorithmic forms of time to project notions of intelligent action that can be understood across human and nonhuman subjects.

\section{Notes}

1. Mary Ann Doane, The Emergence of Cinematic Time: Modernity, Contingency, the Archive (Cambridge, MA: Harvard University Press, 2002), 9.

2. Paul Virilio, Politics of the Very Worst, trans. Michael Cavaliere, ed. Sylvère Lotringer (Los Angeles: Semiotext(e), 1999). Grant Bollmer provides an excellent analysis of Virilio's ideas relative to nineteenth century technics in Grant Bollmer, Materialist Media Theory: An Introduction (New York: Bloomsbury, 2019).

3. Wolfgang Ernst, Digital Memory and the Archive, ed. Jussi Parikka (Minneapolis: University of Minnesota, 2013), 100.

4. Mark B. N. Hansen, Feed-Forward: On the Future of Twenty-First-Century Media (Chicago: University of Chicago Press, 2015), 5.

5. Hansen, Feed-Forward, 58 .

6. See Matthew G. Kirschenbaum, Mechanisms: New Media and the Forensic Imagination (Cambridge, MA: MIT Press, 2008); Nick Montfort, "Continuous Paper: The Early Materiality and Workings of Electronic Literature," paper presented at the Modern Language Association conference, Philadelphia, PA, December 2004, http://nickm.com/writing/essays/continuous_paper_mla.html; Tung-Hui Hu, A Prehistory of the Cloud (Cambridge, MA: MIT Press, 2015); Andrew R. Johnston, "Models of Code and the Digital Architecture of Time," Discourse: Journal for Theoretical Studies in Media and Culture 37, no. 3 (Fall 2015): 221-246. 
7. $\quad$ See Wolfgang Ernst, "Media Archaeology: Method and Machine versus History and Narrative of Media," in Media Archaeology: Approaches, Applications, and Implications, eds. Erkki Huhtamo and Jussi Parikka (Berkeley: University of California Press, 2011), 239-255; Bruno Latour, Pandora's Hope: Essays on the Reality of Science Studies (Cambridge, MA: Harvard University Press, 1999).

8. See James J. Hodge, Sensations of History: Animation and New Media Art (Minneapolis: University of Minnesota Press, 2019); Shane Denson, Discorrelated Images (Durham: Duke University Press, 2020); Adam Hart, Monstrous Forms: Moving Image Horror across Media (Oxford: Oxford University Press, 2019).

9. Wolfgang Ernst, Chronopoetics: The Temporal Being and Operativity of Technological Media, trans. Anthony Enns (London: Rowman \& Littlefield, 2016), 205.

10. See Ernst, "Media Archaeology: Method and Machine," 239.

11. Alvin Toffler, Future Shock (New York: Random House, 1970).

12. See especially Pamela Lee, Chronophobia: On Time in the Art of the 1960 s (Cambridge, MA: MIT Press, 2004).

13. For more on wonder in animation, see Alan Cholodenko, "Introduction" to The Illusion of Life: Essays on Animation, ed. Alan Cholodenko (Sydney: Power Publications in association with the Australian Film Commission, 1991), 9-36; Tom Gunning, "Animating the Instant: The Secret Symmetry between Animation and Photography," in Animating Film Theory, ed. Karen Beckman (Durham, NC: Duke University Press, 2014), 37-53; Colin Williamson, Hidden in Plain Sight: An Archaeology of Magic and the Cinema (New Brunswick, NJ: Rutgers University Press, 2015).

14. Ernst, Chronopoetics, 4.

15. For more on the collapse of interest, funding, and research in AI from this time, see Daniel Crevier, AI: The Tumultuous Search for Artificial Intelligence (New York: Basic Books, 1993).

16. Volodymyr Mnih, Koray Kavukcuoglu, David Silver, Andrei A. Rusu, Joel Veness, Marc G. Bellemare, Alex Graves, Martin A. Riedmiller, Andreas Fidjeland, Georg Ostrovski, Stig Petersen, Charles Beattie, Amir Sadik, Ioannis Antonoglou, Helen King, Dharshan Kumaran, Daan Wierstra, Shane Legg and Demis Hassabis. "Human-Level Control through Deep Reinforcement Learning," Nature 518 (2015): Methods.

17. Terrence J. Sejnowski, The Deep Learning Revolution (Cambridge, MA: MIT Press, 2018), 171-172.

18. See D.H. Hubel and T.N. Wiesel, "Shape and Arrangement of Columns in Cat's Striate Cortex," The Journal of Physiology 165.3 (1963): 559-68; David H. Hubel, Eye, Brain, and Vision (New York: W.H. Freeman, 1988).

19. Hubel, Eye, Brain, and Vision, 131.

20. Yann LeCun, Léon Bottou, Yoshua Bengio, Patrick Haffner, "Gradient-Based Learning Applied to Document Recognition," Proceeding of the IEEE 86.11 (November 1998): 2283-2284. 
21. Mnih, et al., "Human-Level Control," 529.

22. See Frank Rosenblatt, "The Perceptron: A Perceiving and Recognizing Automaton," Report 85-60-1, Cornell Aeronautical Laboratory (Buffalo, New York: 1957); and Frank Rosenblatt, "The Perceptron: A Probabilistic Model for Information Storage and Organization in the Brain," Psychological Review 65 , no. 6 (1958): 386-408.

23. "New Navy Device Learns by Doing: Psychologist Shows Embryo of Computer Designed to Read and Grow Wiser," New York Times, 8 July 1958, 25.

24. Frank Rosenblatt, "The Perceptron: A Probabilistic Model for Information Storage and Organization in the Brain," quoted in Rudolf Seising, "The Emergence of Fuzzy Sets in the Decade of the Perceptron-Lotfi A. Zadeh's and Frank Rosenblatt's Research Work on Pattern Classification," Mathematics 6, no. 7 (2018): 110.

25. Sejnowki, 47 .

26. Mnih, et al., "Human-Level Control," 532.

27. Stephanie Boluk and Patrick LeMieux, Metagaming: Playing, Competing, Spectating, Cheating, Trading, Making, and Breaking Videogames (Minneapolis: University of Minnesota Press, 2017), 8.

28. Patrick LeMieux, "From NES-4021 to moSMB3.wmv: Speedrunning the Serial Interface," Eludamos: Journal for Computer Game Culture 8, no. 1 (2014): 12.

29. Mnih, et al., "Human-Level Control," 531.

30. Karin Knorr Cetina, "Objectual Practice" in The Practice Turn in Contemporary Theory, ed. Theodore R. Schatzki, Karin Knorr Cetina, and Eike von Savigny (London and New York: Routledge, 2001), 190.

31. Lisa Gitelman, Paper Knowledge: Toward a Media History of Documents (Durham: Duke University Press, 2014), 1.

32. Hodge, Sensations of History, 16.

33. For details about DeepMind's use in Starcraft II see Oriol Vinyals, Timo Ewalds, Sergey Bartunov, Petko Georgiev, Alexander Sasha Vezhnevets, Michelle Yeo, Alireza Makhzani, Heinrich Küttler, John Agapiou, Julian Schrittwieser, John Quan, Stephen Gaffney, Stig Petersen, Karen Simonyan, Tom Schaul, Hado van Hasselt, David Silver, Timothy P. Lillicrap, Kevin Calderone, Paul Keet, Anthony Brunasso, David Lawrence, Anders Ekermo, Jacob Repp and Rodney Tsing, "StarCraft II: A New Challenge for Reinforcement Learning," ArXiv abs/1708.04782 (2017): n. pag.; Vinicius Zambaldi, David Raposo, Adam Santoro, Victor Bapst, Yujia Li, Igor Babuschkin, Karl Tuyls, David Reichert, Timothy Lillicrap, Edward Lockhart, Murray Shanahan, Victoria Langston, Razvan Pascanu, Matthew Botvinick, Oriol Vinyals, Peter Battaglia, "Deep Reinforcement Learning with Relational Inductive Biases," International Conference on Learning Representations, 2019.

34. See, for example, Jonathan Sterne, The Audible Past: The Cultural Origins of Sound Reproduction (Durham: Duke University Press, 2003) and David Parisi, Archaeologies of Touch: Interfacing with Haptics from Electricity to Computing (Minneapolis: University of Minnesota Press, 2018). 
35. Bernard Dionysius Geoghegan, "An Ecology of Operations: Vigilance, Radar, and the Birth of the Computer Screen," Representations 147, no. 1 (Summer 2019): 59-95.

\section{About the Author}

Andrew R. Johnston is Associate Professor in the Department of English, the Film Studies Program, and the Communication, Rhetoric, and Digital Media Program at North Carolina State University. His book Pulses of Abstraction: Episodes from a History of Animation (University of Minnesota Press, 2020) examines abstract animation in cinema and computational media from the 1950s-70s. 